\title{
Approaches for short-term photovoltaic energy production forecast in Russian climate conditions
}

\author{
Semen Frid ${ }^{1, *}$, Sophia Kiseleva ${ }^{2}$, Natalya Lisitskaya $^{1}$ and Alexander Mordinsky ${ }^{1}$ \\ ${ }^{1}$ Joint Institute for High Temperatures of the Russian Academy of Sciences (JIHT RAS), 125412 13/2 Izhorskaya, Moscow, Russia \\ ${ }^{2}$ Lomonosov Moscow State University, 1199911 Leninskie Gory, Moscow, Russia
}

\begin{abstract}
Different techniques for short-term energy production by photovoltaic solar parks (PVSP) were considered taking into account specific features of Russian energy market. Based on theoretical simulation using numerical weather prediction results and experimental data it has been shown, that even for low precision techniques their application allows to improve economics of PVSP's operation. With a decrease in the forecast error by 1.5-2 times, the economic losses due to the grid dispatch schedule deflection decrease by $5-9$ times.
\end{abstract}

\section{Introduction}

Photovoltaic systems, due to the solar radiation incidence irregularity, cannot guarantee the electrical power delivery to grid at a specified time. Therefore, the forecast of energy entering the grid from photovoltaic solar parks (PVSP) is an actual research direction. Due to the PVSPs amount rapid growth a number of countries in recent years initiated research activity in the field of short-term forecasting for the PVSPs power output [1, 2]. Particular attention is paid to a day ahead forecasting with hourly resolution. A demand for such time range forecast is determined by the sustainable grid operation and the current procedure for electric power trading on the wholesale market for many countries of the world, including Russia. However, the forecast accuracy requirements are different due to different market trading system regulations. So, the national standard of China [3] requires PVSPs to provide energy production forecast for the day ahead with 15 minute detailing.

Since 2013, in the Russian Federation, the PVSPs has been subsidized by the state. The investor's costs for the PVSPs construction are partially or fully compensated under the following conditions:

- achievement of the solar power plant installed capacity factor average annual value not less than 10.5\%;

- meeting the requirements for the PVSPs components production localization;

- the minimum PVSP construction cost declared at the competition.

As a result, the main sources for investor payments recovery are payments for the power (not energy) supply. The sale of electric energy generated by the PVSP is secondary from the object's payback point of view. Such a system differs from many foreign countries, using feed-in tariff for renewables.
The specific of state support measures in Russia determines a special approach to formulating the forecast methods problem. It is important to develop a forecasting method that would be sufficient to minimize economic losses from forecast inaccuracies. In the Russian Federation, the Rules of the Wholesale Electricity and Power Market (WECM) [4] establish the permissible actual hourly power output deviations from the volume declared for the day ahead for PVSP. There are no regulatory methods for predicting the PVSP power output. In the Russian scientific literature shortterm PVSP forecasting productivity within the framework of the functioning of the energy market has not received wide development at the moment. A small number of works have been published, carried out mainly in various universities as a part of educational process. However, this issue is becoming more and more important for PVSP operators (Hevel Group, Solar Systems Group, etc.). This paper briefly consider the main approaches to forecasting the production of briefly consider the main approaches to forecasting the production of photovoltaic plants in connection with their grid operation.

\section{PVSP energy production forecasting techniques}

The methods for the PVSP generation developed to date can be classified according to various criteria:

- predictive operations sequences (direct, i.e. without predicting the solar radiation sum for the period of time, indirect, hybrid);

- the time horizon.

The forecast methods and initial data choice are mainly determined by the forecast horizon:

- statistical models and machine learning methods (for forecasting period from several minutes to an hour);

* Corresponding author: $\underline{\text { s frid@oivtran.ru }}$ 
- meteorological models based on satellite observations and sky image cameras (for several hours);

- numerical weather prediction (NWP, for the 6-hours horizon or more).

Since the energy production variations for an individual photovoltaic module (PVM) are significantly greater than the PVSPs group performance, the forecast error is also significantly reduced when switching from forecasting the production of an individual PV module to forecasting for a PVSPs group.

In addition to the deterministic ones, probabilistic forecasts are being developed, which determine the range of probable values of solar radiation input, solar power generation, etc., as well as each forecast probability. Probabilistic forecasts can provide data for grid operators in case of electric power reservation considering uncertainty in the PVSPs generation, but in Russia these data are usually not used. For deterministic and probabilistic forecasts, different accuracy metrics are used.

Among the most widely used and developed techniques, the following deterministic methods for the PVSPs performance forecasting should be considered:

- persistent models are the simplest models, considering as the primary forecasting stage; they assume the constancy of current conditions for which the PVSPs performance forecast is made;

- PV-forecast, which is based on the PVSP performance calculation based on a numerical weather prediction, first of all, in terms of the solar radiation sum in considered time period, and the main error of this method is determined precisely by the NWP error;

- machine learning methods.

The best results in terms of forecast accuracy are provided by hybrid methods that combine the latter two approaches.

An important element of the PV forecast is PVSPs parameters involving (size, power, orientation of the PV module in space, power and efficiency of the inverter, the age of the system), as well as local physical and geographical conditions.

\section{NWP data sources}

Numerical weather prediction is the result of aerodynamic processes and atmospheric energy flows simulation. The problem complexity and the need for real-time simulation necessitate the use of supercomputers for NWP and the impossibility of such simulation inside of installing company. There are several atmospheric models and NWP algorithms are implemented on their basis in national and international meteorological centers. In Russian practice, the meteorological parameters forecast is carried out by numerical simulation in the COSMO-Ru short-term weather forecast system. The calculation is performed by specialists of the Hydrometcenter of Russia 4 times a day. During such calculation, the fluxes of direct and diffuse solar radiation are derived, however, these data are considered as auxiliary and is not accumulated in the archive database. Access to them is difficult. In foreign practice, when predicting the generation of PV power plants, the WRF and IFS models are used to forecast the solar radiation income. The first is based on the American regional model WRF-ARW and is in little use for Russian conditions. The European Center for Medium-Range Weather Forecasts (ECMWF) IFS weather forecast is an expensive commercial product. There are also several commercial services that declare the forecast provision with a horizon of up to 10 days for the solar radiation sums and the generation of PVSPs [5]. Two of them, Solcast [6] and Solargis [7], provide noncommercial trial use. The first one can execute up to 1000 queries, declared spatial resolution is $1-2 \mathrm{~km}$. The Solargis trial period is 3 months, the spatial resolution of the solar radiation forecast is $33-55 \mathrm{~km}$.

Among the freely available sources of NWP information [8], the ICON model [9] from the German Weather Service (DWD) and the Max-Planck Institute for Meteorology in Hamburg (MPI-M) is worth mentioning. Predictive fields of ICON are used in the COSMO-Ru model as initial and boundary conditions. The grid spacing of the global ICON model is $13 \mathrm{~km}$, the European one - ICON-EU - $6.5 \mathrm{~km}$. Calculation by ICON is performed 4 times a day for 120-180 hours in advance and 4 more times - for 30 hours. The calculation results are freely available via http or ftp protocols, and the license allows any use of them providing a link to the data source. Calculations results using the probabilistic ICON-EPS and ICON-EU-EPS models are laid out in this database.

Price orders, according to the wholesale electricity and power market rules are submitted before 13:30 Moscow time for the next day. So taking into account the delay in the calculation results on servers posting, the results of the NWP performed at 6 hours UTC with a forecasting horizon of 40 hours are required. An array of ICON, ICON-EU and ICON-EU-EPS forecast data has been accumulated since January 2020, as well as Solcast data for 4 points in Russia since November 2019.

\section{Forecasting error and its influence on PVSP indicators}

In accordance with the WECM Rules [4], PVSPs power deviations from the price order for the day ahead are not penalized in the range of $\pm 10 \%$ of the installed PVSP power. Losses from PVSP dispatch schedule deviations are generated in two cases. First case is the lost profit when the actual production exceeds the allowable range of generation deviations. The second is concerned with losses caused by the accrual of the negative balancing market imbalance on the volume.

Lost profit arises due to the fact that excess energy is paid at a rate of $1 \mathrm{r}$./MWh, and for each megawatt-hour generated, the PVSP loses $\left(C_{s r w}-1\right)$, where $C_{s r w}$ is the energy market price for the day ahead. Losses from negative imbalance are due to the difference between the $C_{s r w}$ and the price indicator of the balancing market. Both price parameters are determined for each gridconnection point and change every hour, i.e. are unique for each PVSP. 
To analyse the forecast error impact on the PVSP economic performance, we used data on price indicators and the output for two objects from the Solar Systems Group: PVSP Zavodskaya (installed capacity $15 \mathrm{MW}$, forecast periods - February, July, November 2019) and PVSP "Samarskaya" (installed capacity 75 MW, November 2019). The calculation of losses from dispatch schedule deviations was carried out both for the predicted and actual production values given in the price bids, and for model cases corresponding to a predicted value deviation from the actual data decrease by $1.5 ; 2$ and 4 times. The mean square error RMSE was chosen as a forecast error quantity measure. The results are illustrated by Fig. 1. The calculation showed that the forecast error reduction by a factor of 2 leads to losses decrease from the dispatch schedule non-fulfillment by 5-9 times. So it is necessary to reduce the forecast error by 1.5-2 times to reduce losses from PVSP dispatch schedule non-fulfillment to reasonably low limits.

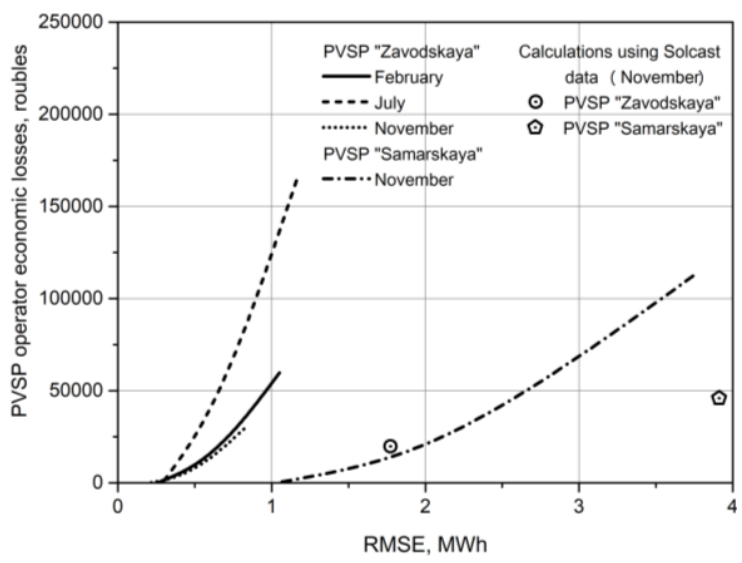

Fig. 1. PVSP operator economic losses dependence from the forecasting mean square error for Zavodskaya and Samarskaya PVSPs.

Computational studies of the NWP data error influence the PVSPs simulated operation were also carried out. For this purpose, using the Solcast service, Zavodskaya and Samarskaya solar power plants operation in November 2019 have been simulated in the TRNSYS system for renewable energy systems dynamic modeling [10]. The results are shown as separate points in Fig. 1. Despite the greater than in expert forecasting, the value of the mean square forecast error, losses from the PVSP dispatch schedule non-fulfillment turned out to be 2.5-3 times lower. This is due to the fact that NWP gives a more "uniform" forecast, without sharp peaks. These peaks make the greatest contribution to losses from the dispatch schedule non-fulfillment. So NWP use allows to reduce losses even without the forecast error reduction. At the same time, the mean square forecast error value, normalized to the nominal capacity, was $5.2 \%$ for the Samarskaya PVSP, and about $12 \%$ for the Zavodskaya PVSP.

Similar values were obtained by comparing the numerical simulation of model grid-tie system hourly operation according to ICON, ICON-EU-EPS and Solcast forecasts results with its actual values in the period from July 11 to August 12, 2020. The model system called RESEN-12 is equipped with $36 \mathrm{MSW}-180$ PV panels located in JIHT RAS. The root mean square error normalized to the installed power of the system $(6.48 \mathrm{~kW})$ is $12.3 \%, 12.7 \%$ and $13.4 \%$, respectively for different data sources. At the same time, the correlation between the calculated and experimental output is weak, taking into account data from Fig. 2 scatter diagram. The scatterplots for the other forecast services used have a close view.

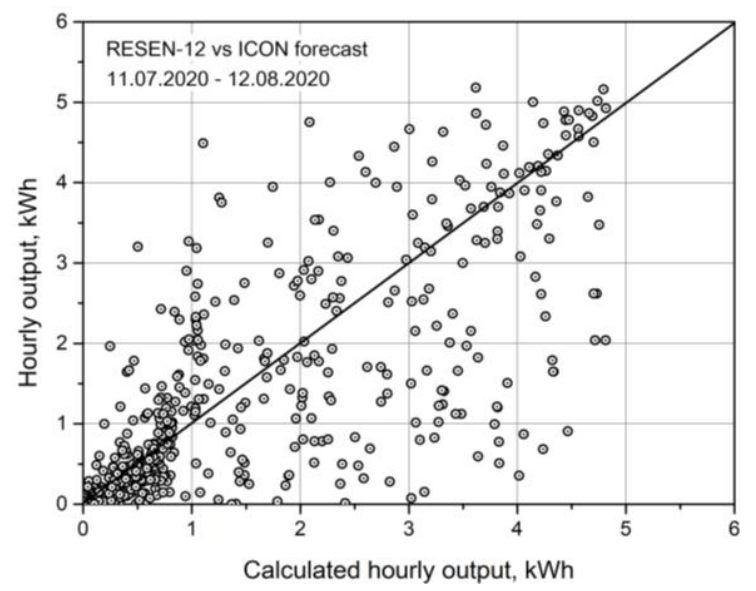

Fig. 2. RESEN-12 hourly production scattering plot.

The calculation results, power output data collected from RESEN-12 and the Solar Systems Group of Companies PVSPs operation results allow us to assume that, despite the rather large production forecast error obtained directly from PVSP operation simulation (using numerical weather forecast data); such a forecast allows reducing losses from the dispatch schedule nonfulfillment comparing to expert forecast. Such a forecast can be used as a first approximation and, from this point of view, can be considered persistent. At the same time, the NWP error for freely available and commercial services does not fundamentally differ. Further PVSP output forecast refinement is necessary only to help to reduce economic losses, and should be aimed at minimizing them. It can be carried out by the regression implemented by machine learning methods, taking into account a wider list of climatic conditions for the PVSPs operation.

\section{Conclusion}

PV systems power output prediction associated with the variable solar radiation arrival on the earth's surface makes more suitable PV grid-tie applications. The specificity of the Russian electric power market is that the main income received by the PVSP operator arises from the compensation payments for capacity. Electric energy is sold on the day-ahead futures market, and market rules do not impose strict requirements on forecast accuracy else. Over the past 10-20 years, new PV systems power output forecasting methods have been developed, but a day-ahead forecast requires numerical weather prediction data. 
Based on the data provided by commercial services and freely available forecasts, a local archive of hourly incident solar radiation sums and other meteorological parameters forecasting for a number of points in Russia has been created. The short-term PVSP generation forecasting technique in the form of direct numerical PVSP operation simulation is based on numerical weather prediction data. Despite relatively low accuracy of this technique, it improves the PVSP economic indicators in comparison with the expert forecast. To reduce losses from the PVSP dispatch schedule nonfulfillment to reasonably low limits, it is necessary to reduce the forecast error by $1.5-2$ times.

The study was financially supported by the Russian Foundation for Basic Research in the scope of project no 19-08-00877.

\section{References}

1. J. Kleissl, Solar Energy Forecasting and Resource Assessment (Academic Press, 2013)

2. J. Antonanzas, N. Osorio, R. Escobar, R. Urraca, F.J. Martinez-de-Pison, F. Antonanzas-Torres, Solar Energy 136, 78-111 (2016)

3. T. Reindl, W. Walsh, Z. Yanqin, M. Bieri, Energy Procedia 130, 130-138 (2017)
4. Regulation for determining the volumes, initiatives and cost of deviations. Appendix 12 to the Agreement on Joining the Wholesale Market Trading System, Association "Nonprofit Partnership Council for Organizing Efficient System of Trading at Wholesale and Retail Electricity and Capacity Market”, url: https://k.np-sr.ru/sites/default/files/ sr_regulation/reglaments/r12_01012018_ 24012018.pdf (in Russian)

5. PV Energy forecasts, url: https://photovoltaicsoftware.com/solar-tools/pv-energy-forecasts

6. Solar Forecasting \& Solar Irradiance Data, url: https://solcast.com

7. Solar radiation and PV power forecast, Solargis, url: https://solargis.com/products/forecast/overview

8. Free NWP data, IEA wind task 36, url: https://www.ieawindforecasting.dk/free-nwp-data

9. Wetter und Klima, Deutscher Wetterdienst, Our services, NWP forecast data, url: https://www.dwd.de/EN/ourservices/ nwp_forecast_data/nwp_forecast_data.html

10. TRNSYS - Transient System Simulation Tool, url: http://trnsys.com 